\title{
Tumor protein p53 mutation in archived tumor samples from a 12-year survivor of stage 4 pancreatic ductal adenocarcinoma may predict long-term survival with DeltaRex-G: A case report and literature review
}

\author{
MICHAEL A. MORSE ${ }^{1}$, SANT P. CHAWLA ${ }^{2}$, TERENCE Z. WONG ${ }^{1}$, \\ HOWARD W. BRUCKNER ${ }^{3}$, FREDERICK L. HALL ${ }^{4}$ and ERLINDA M. GORDON ${ }^{2,4,5}$ \\ ${ }^{1}$ Medical Oncology, Duke University Medical Center, Durham, NC 27710; \\ ${ }^{2}$ Cancer Center of Southern California, Santa Monica, CA 90403; ${ }^{3}$ Bruckner Oncology, Bronx, NY 10469; \\ ${ }^{4}$ Delta Next-Gene, LLC; ${ }^{5}$ Aveni Foundation, Santa Monica, CA 90405, USA
}

Received December 3, 2020; Accepted June 25, 2021

DOI: $10.3892 / \mathrm{mco} .2021 .2348$

\begin{abstract}
DeltaRex-G is a replication-incompetent amphotropic murine leukemia virus-based retroviral vector that displays a collagen-matrix-targeting decapeptide on its surface envelope protein, gp70, and encodes a cytocidal 'dominant negative', i.e. a truncated construct of the executive cyclin G1 (CCNGI) oncogene. DeltaRex-G inhibits the $C C N G 1$ function of promoting cell competence and survival through the commanding $C C N G 1 /$ cyclin-dependent kinase (CDK)/Myc/mouse double minute 2 homolog $(\mathrm{Mdm} 2) / \mathrm{p} 53$ axis. In 2009, DeltaRex-G was granted Fast Track designation from the US Food and Drug Administration for the treatment of pancreatic cancer. In 2019, the results of a phase 1/2 study that used DeltaRex-G as monotherapy for stage 4 chemotherapy-resistant pancreatic ductal adenocarcinoma (PDAC) were published. A unique participant of the aforementioned phase $1 / 2$ study is now an 84-year-old Caucasian woman with chemoresistant PDAC who was treated with DeltaRex-G, $3 \times 10^{11}$ colony forming units (cfu)/dose, 3 times/week for 4 weeks with a 2 -week rest period, for 1.5 years. During the treatment period, the patient's tumors in the liver, lymph node and peritoneum exhibited progressive decreases in size, which were accompanied by a reduction and normalization of serum carbohydrate antigen 19-9 levels, and the patient achieved complete remission after 8 months of DeltaRex-G therapy with minimal side effects (grade 2 fatigue). Henceforth, the patient has been in remission for 12 years with no evidence
\end{abstract}

Correspondence to: Dr Erlinda M. Gordon, Cancer Center of Southern California, 2811 Wilshire Boulevard, Suite 414, Santa Monica, CA 90403, USA

E-mail: erlinda.gordon@gmail.com

Key words: CCNG1 inhibitor, TP53, cancer gene therapy, pancreatic adenocarcinoma, case report of disease, no late therapy-related adverse events, and no further cancer therapy following DeltaRex-G treatment. The present study reports a mutation of tumor protein p53 (TP53) $(\mathrm{G} 199 \mathrm{~V})$ found retrospectively in the patient's archived tumor samples. TP53 is a well-characterized tumor suppressor gene, and a critical regulatory component of the executive $C C N G 1 / \mathrm{CDK} / \mathrm{Myc} / \mathrm{Mdm} 2 / \mathrm{p} 53$ axis, which regulates proliferative cell competence, DNA fidelity and survival. Studies are underway to determine whether TP53 mutations in pancreatic cancer can help identify a subset of patients with advanced metastatic cancer with an otherwise poor prognosis who would respond favorably to DeltaRex-G, which would broaden the treatment options for patients with otherwise lethal PDAC.

\section{Introduction}

Metastatic pancreatic ductal adenocarcinoma (PDAC) is a serious disease with a 5-year survival rate of $3 \%$ (1). Therefore, clinical trials using innovative therapies are urgently required. In recent years, molecular profiling and next-generation sequencing of archived or resected tumor samples have been developed, and certain genetic mutations in tumors have predicted favorable responses to gene-targeted inhibitor therapies (2). A unique patient with chemoresistant PDAC metastatic to the liver, lymph nodes and peritoneum is reported in the present study because she participated in a phase I/II clinical trial that used DeltaRex-G, the first-in-human intravenously (i.v.) administered tumor-targeted gene therapy approach to stage 4 pancreatic cancer (3), has survived beyond the median survival time of 8 months reported for the optimal first-line therapy (gemcitabine and nab-paclitaxel) (4) and beyond the 5-year survival time reported for metastatic PDAC (1), with no evidence of cancer or delayed therapy-related adverse events for $>12$ years. Furthermore, her tumor harbored a genetic mutation that could favorably broaden the limited range of treatments available for the otherwise lethal prognosis of stage 4 pancreatic cancer. 
DeltaRex-G (formerly Mx-dnG1, dnG1 or Rexin-G) is a replication-incompetent tumor-targeted retroviral vector that displays a collagen matrix (Signature, SIG)-binding decapeptide for targeting anaplastic collagenous (SIG) proteins exposed by the invading tumor and encodes a dominant negative mutant construct of the cyclin G1 (CCNGI) gene that is devoid of its $\mathrm{N}$-terminus and the first two helical segments ( $\alpha 1$ and $\alpha 2)$ of the definitive cyclin (proteolytic processing). The cytocidal dnG1 protein, which induces apoptosis in proliferating cells, retains the cyclin-dependent kinase (CDK) contact points (helices $\alpha 3^{*}$ and $\alpha 5^{*}$ ) and the structural domains for serine/threonine protein phosphatase subunit designated $2 \mathrm{~A}$ (PP2A), $\beta^{\prime}$ and Mdm2 binding, ultimately blocking CCNG1 function and proliferative cell competence and survival through the commanding $C C N G 1 / \mathrm{CDK} / \mathrm{Myc} / \mathrm{Mdm} 2 / \mathrm{p} 53$ axis (5).

\section{Case report}

Patient information and clinical findings. In late 2006 , the patient was initially diagnosed with localized, poorly differentiated PDAC, underwent a Whipple's procedure with postoperative radiation therapy, and received fluorouracil chemoradiotherapy and external beam radiation, followed by gemcitabine. In 2008, the patient presented with hepatic and lymph node metastases, and peritoneal carcinomatosis based on abnormalities on the fluorodeoxyglucose-positron emission tomography scan and elevated serum carbohydrate antigen (CA)19-9 levels. At that time, the patient refused further chemotherapy and decided to participate in a phase I/II study using DeltaRex-G, a tumor-targeted retrovector encoding and expressing a truncated cytocidal construct of the CCNG1 oncogene, which blocks $C C N G 1$ function in the malignant cell cycle (3).

Therapeutic intervention. The advanced phase I/II clinical trial (NCT00504998) was a dose-seeking study that incorporated a modified cohort-of-3 design (3). Increasing doses of DeltaRex-G [1.0-3.0 x10 11] colony-forming units (cfu)/dose] were administered i.v. two/three times per week for 4 weeks with a 2 -week rest period, which comprised one treatment cycle. Treatment cycles were repeated if grade $\leq 1$ toxicity was observed. Treatment response was evaluated based on the Response Evaluation Criteria in Solid Tumors (v1.0). Safety and efficacy analyses were conducted by the Site Principal Investigators (clinical sites: Santa Monica, Brooklyn and Durham, USA). The clinical protocol was reviewed and approved by the Western Institutional Review Board (Olympia, WA, USA). The patients were recruited on a first-come, first-served basis, and written informed consent was obtained from each patient at the time of enrollment. All personnel who handled and disposed of the vector complied to biosafety level 2 requirements in accordance with the NIH Guidelines for Research Involving Recombinant or Synthetic Nucleic Acid Molecules.

Follow-up and outcomes. The patient received dose level 3, which consisted of $3 \times 10^{11} \mathrm{cfu}$ DeltaRex-G/dose three times per week for 4 weeks with a 2 -week rest period (one treatment cycle) for 1.5 years. The progressive reduction in the sum of

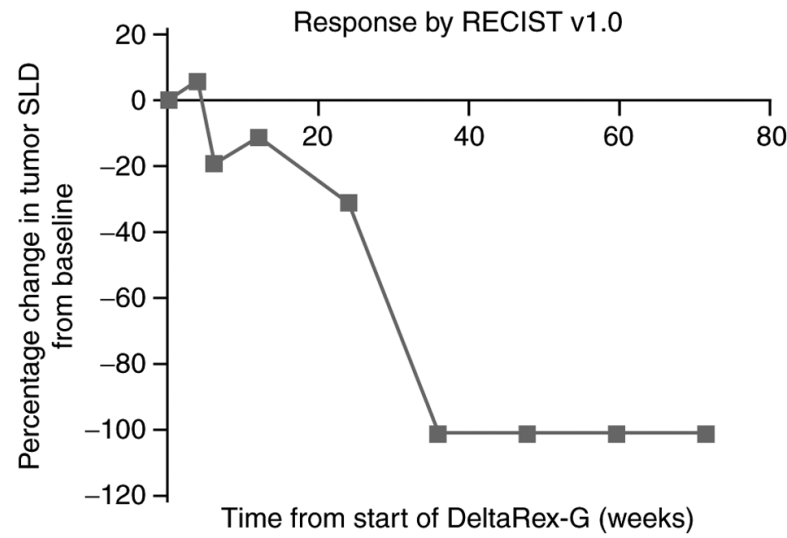

Figure 1. Progressive reduction in tumor burden according to RECIST v1.0 during treatment with DeltaRex-G in a patient with metastatic pancreatic adenocarcinoma. Change in tumor SLD from baseline (\%) is plotted on the vertical axis as a function of time during treatment with DeltaRex-G at $3 \times 10^{11} \mathrm{cfu} /$ day three times a week, plotted on the horizontal axis. SLD, sum of the longest diameters; RECIST, Response Evaluation Criteria in Solid Tumors; cfu, colony forming units.

the longest tumor diameters over time is shown in Fig. 1. The patient achieved complete remission with minimal toxicity (grade 2 fatigue) after 8 months of therapy (Fig. 2). She received no further treatment following the completion of the study and has achieved a sustained remission with normal serum CA19-9 levels $(32 \mathrm{ng} / \mathrm{ml})$, and no late-onset treatment-related adverse events as of the last follow-up in April 2021.

Diagnostic assessment. 'FoundationOne ${ }^{\circledR} \mathrm{CDx}$ is performed exclusively as a laboratory service using DNA extracted from formalin-fixed, paraffin-embedded (FFPE) tumor samples. The assay involves a single DNA extraction from routine FFPE biopsy or surgical resection specimens; 50-1,000 ng DNA then undergoes whole-genome shotgun library construction and hybridization-based capture of all coding exons from 309 cancer-related genes, one promoter region, one non-coding RNA and selected intronic regions from 34 commonly rearranged genes, 21 of which also include the coding exons. In total, the assay detects alterations in a total of 324 genes. Using the Illumina ${ }^{\circledR}$ HiSeq 4000 platform (Illumina, Inc.), hybrid capture-selected libraries are sequenced to high uniform depth (targeting $>500 \mathrm{X}$ median coverage with $>99 \%$ of exons at a coverage of $>100 X)$. Sequence data are then processed using a customized analysis pipeline designed to detect all classes of genomic alterations, including base substitutions, indels, copy number alterations (amplifications and homozygous gene deletions), and select genomic rearrangements (e.g., gene fusions)'.

Retrospective RNA sequence analysis of this patient's archived tumor samples, performed by Foundation One ${ }^{\circledR} \mathrm{CDx}$, showed two clinically significant genetic mutations: KRAS proto-oncogene, GTPase (KRAS; G12R) and tumor protein p53 (TP53; G199V). In addition, the patient had simultaneous U2 small nuclear RNA auxiliary factor 1 (U2AF1; S34F) gene expression, which is considered to be involved in epithelial-to-mesenchymal transition (EMT) and increased tumor cell invasion (6). While KRAS and TP53 mutations are frequently found in PDAC (7) and are associated with poor prognosis, these genetic alterations have not, thus far, been targetable. 
A

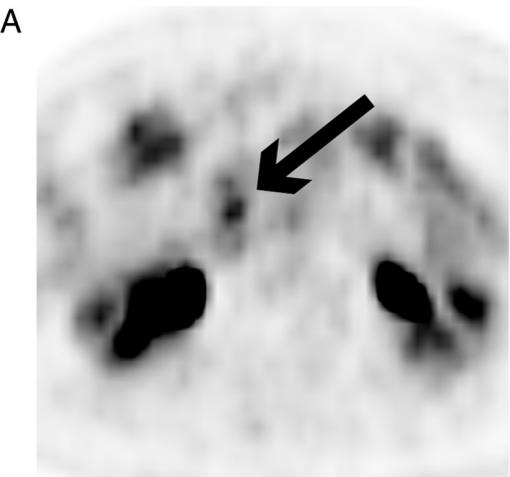

D

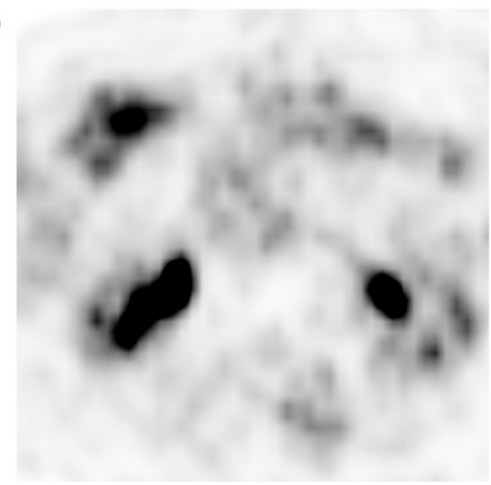

B

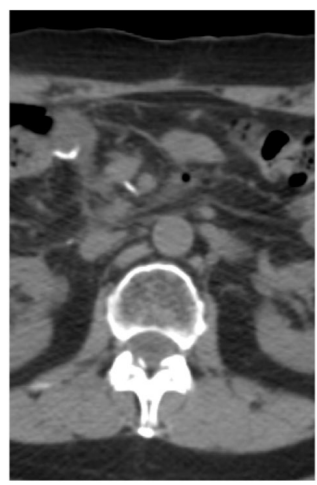

E

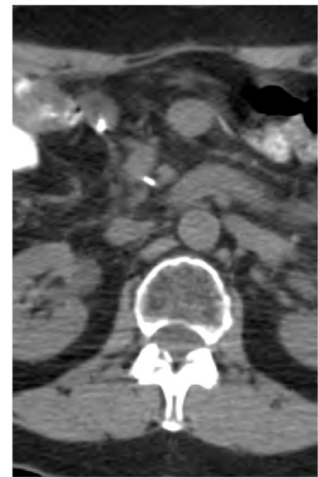

C

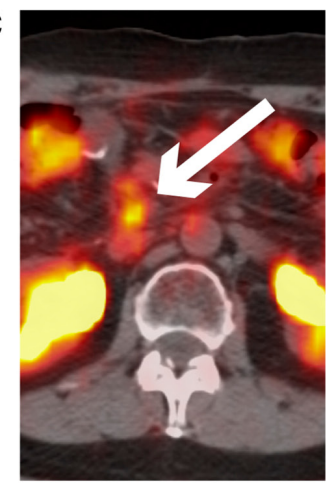

F

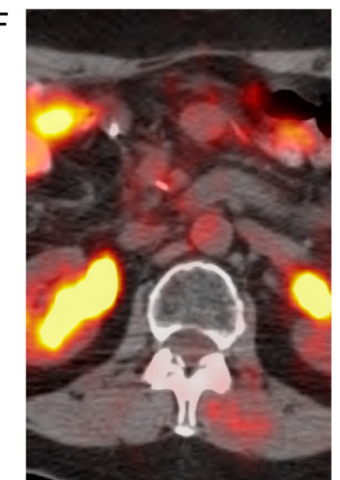

Figure 2. Resolution of FDG-glucose uptake in a metastatic lymph node following DeltaRex-G therapy. (A) Prior to DeltaRex-G treatment, avid uptake of FDG-glucose was observed in the metastatic lymph node by PET alone (arrow). (B) These observations were not readily seen by CT alone. (C) Avid uptake of FDG-glucose was also noted by PET-CT (arrow). (D) After treatment with DeltaRex-G, no FDA-glucose uptake was observed by PET. (E) These observations were not readily seen by CT alone. (F) After treatment with DeltaRex-G, no FDA-glucose uptake was observed by PET-CT. FDG, fluorodeoxyglucose; PET, positron emission tomography; CT, computerized tomography.

\section{Discussion}

DeltaRex-G is an immunologically stealth (repeatedly injectable)retrovector displaying a Signature collagen-matrix-binding targeting peptide on its gp70 Env protein and encoding a cytocidal dominant negative CCNG1 inhibitor gene, which blocks the executive $C C N G 1$ axis. When injected i.v., the DeltaRex-G nanoparticles ( 100 nm in diameter) seek out the tumor and accumulate in the tumor microenvironment (TME), where anaplastic collagenous proteins secreted by tumor-associated fibroblasts (TAFs) constitute an abnormal finding, thus increasing the effective drug concentration in the TME in the vicinity of proliferating cancer cells. The vector then enters the cancer cell and delivers its cytocidal genetic construct into the nucleus of rapidly dividing cancer cells, TAFs and neoangiogenic cells, causing apoptosis by blocking the G1 phase of the cell division cycle (8). The 10 steps of the DeltaRex-G function are shown in Fig. 3.

The discovery that $C C N G 1$ is physically associated with both PP2A and Mdm2, and that this physical association regulates the accumulation and degradation of the $\mathrm{p} 53$ protein, has provided new and important insights into the oncogenic function of $C C N G 1$, and suggests that a major role of $C C N G 1$ is to activate the $\mathrm{Mdm} 2$ oncoprotein to override the cell cycle checkpoint control functions of p53 (8). The loss of p53-mediated tumor suppression in addition to the mutational activation of the KRAS oncogene was found to drive multiple oncogenic signaling cascades (Fig. 4), including mitogen-activated protein kinase, phosphoinositol-3 kinase and transforming growth-factor- $\beta$ pathways governing cancer stem cell survival, proliferation and metastatic behavior (EMT and U2AF1 S34F gene expression). These findings may uncover a potential mechanism for $C C N G 1$-related growth promotion, rather than simply p53-mediated growth arrest (9-11). This hypothesis of the pro-survival and pro-growth function of the CCNGI oncogene is further supported by the reduced incidence of hepatic tumors in $C C N G 1$ knockout mice upon exposure to hepatocarcinogens followed by partial hepatectomy (12). The decrease in tumor predisposition associated with the loss of $C C N G 1$ function during embryogenesis was partially due to a consequential increase in p53 levels and p53 tumor suppressor activity (9). In combination, these findings may provide evidence to support a unifying molecular genetic hypothesis: That the strategic modulation of $C C N G 1$ function(s) observed in the commanding $C C N G 1 / \mathrm{CDK} 2 / \mathrm{Myc} / \mathrm{Mdm} 2 / \mathrm{p} 53$ axis (Fig. 4) may guide the development of novel, precise targeted anticancer agents, such as DeltaRex-G (10), as well as for combinatorial approaches.

Consistent with this hypothesis, MiaPaca-2 cell lines (p.R248W; American Tissue Culture Collection) and human xenograft murine models of pancreatic cancer expressing TP53 hot-spot mutations continue to demonstrate significant sensitivity to CCNG1 inhibitor treatment $(13,14)$. High-level transduction efficiency and cytocidal activity of DeltaRex-G vector have been reported in MiaPaca-2 cells in vitro (14). Furthermore, the systemic delivery of DeltaRex-G was found 


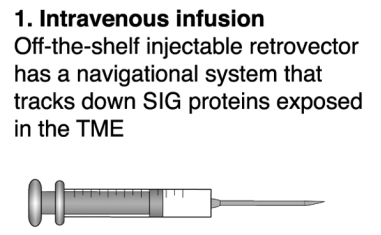

\section{DeltaRex-G mechanism of action}

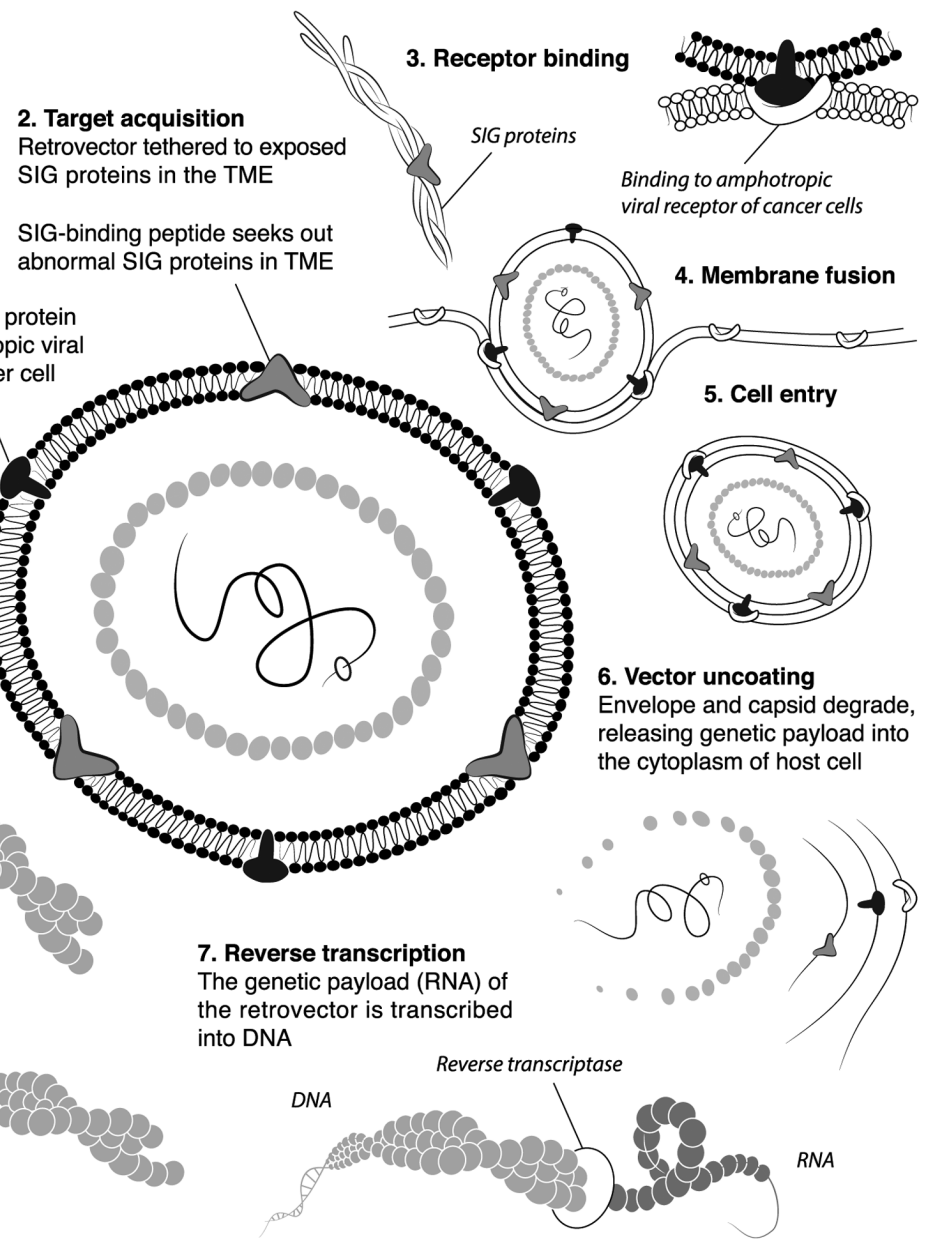

Figure 3. Ten-step illustration of DeltaRex-G mechanism of action. The DeltaRex-G nanoparticle displays a collagen matrix (SIG)-binding peptide derived from coagulation vWF on its gp70 envelope protein. When injected i.v., DeltaRex-G seeks out the tumors and accumulates in cancerous lesions by binding to abnormal collagenous SIG proteins exposed in the TME as a result of tumor invasion. This chimeric retrovector has the innate property of binding to the natural amphotropic viral/cell receptor, fusing, entering, uncoating and integrating randomly into the chromosomes of only actively dividing cells (i.e., cancer cells), sparing normal cells. DeltaRex-G bears a cytocidal CCNG1 inhibitor gene, which causes cell death through apoptosis. CCNG1, cyclin G1; SIG, abnormal signature; vWF, von Willebrand factor; TME, tumor microenvironment.

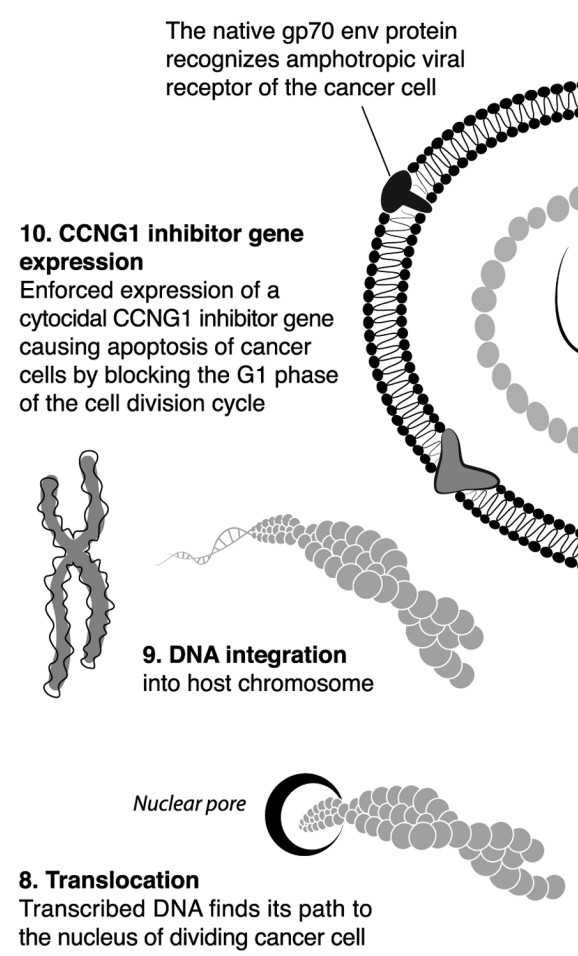

7. Reverse transcription enetic payload (RNA) of DNA

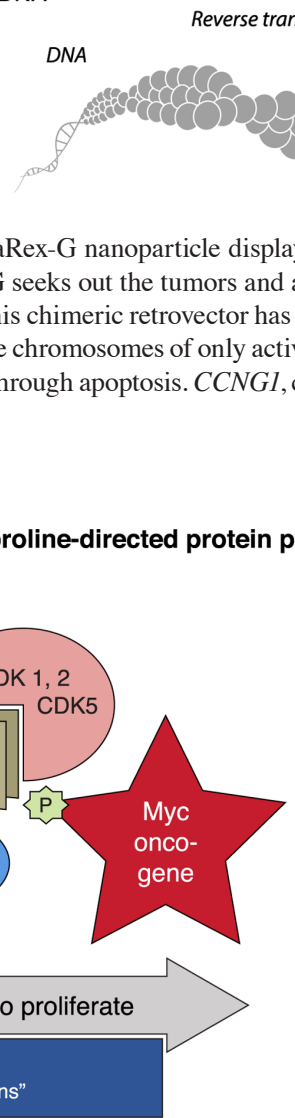

Mitogenic signal transduction via proline-directed protein phosphorylation
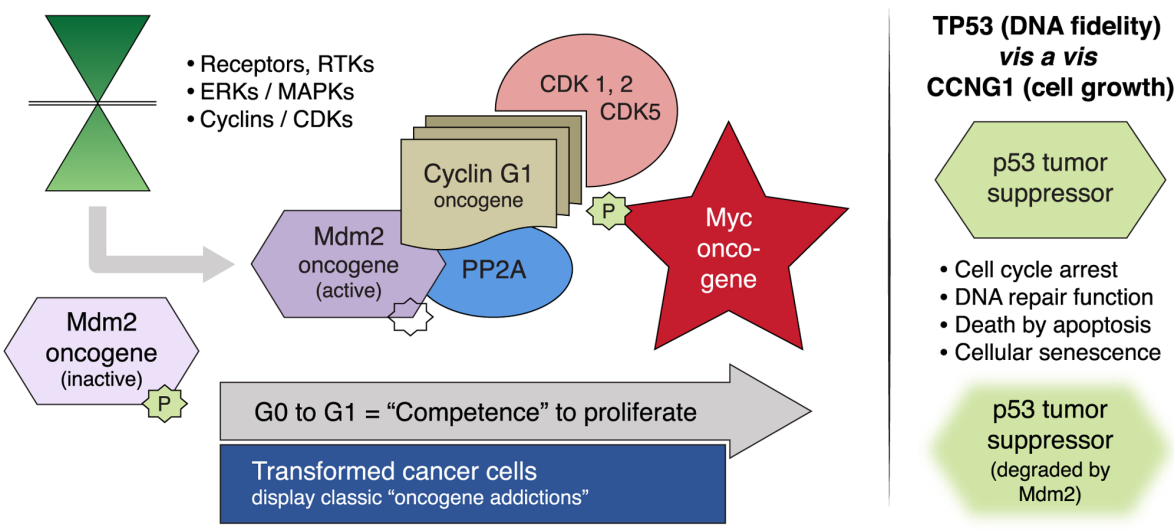

Figure 4. Mitogenic signaling pathways and the human CCNG1 gene. Left panel, RTKs, MAPKs/ERKs and CDK complexes control the progressive phases of the cell division cycle. CCNG1 physically binds to the PP2A to activate a key regulatory oncoprotein, Mdm2. The Mdm2 oncoprotein forms a physical complex with the p53 tumor suppressor, thus inactivating its tumor suppressor function, while also acting as a specific E3 ubiquitin ligase responsible for the ubiquitination and degradation of the p53 tumor suppressor protein. This dephosphorylation event is CCNG1-dependent. CCNG1 also activates CDK5 and CDK1/2 to target/activate the c-Myc oncoprotein. Right panel, TP53 tumor suppressor functions are presented as opposing to the CCNG1 growth-promoting function. CCNG1, cyclin G1; RTK, receptor tyrosine kinase; MAPKs, mitogen-activated protein kinases; ERKs, extracellular-signal-regulated kinases; CDK, cyclin-dependent kinase; PP2A, serine/threonine protein phosphatase subunit designated 2A; Mdm2, mouse double minute 2 homolog; TP53, tumor protein p53. 


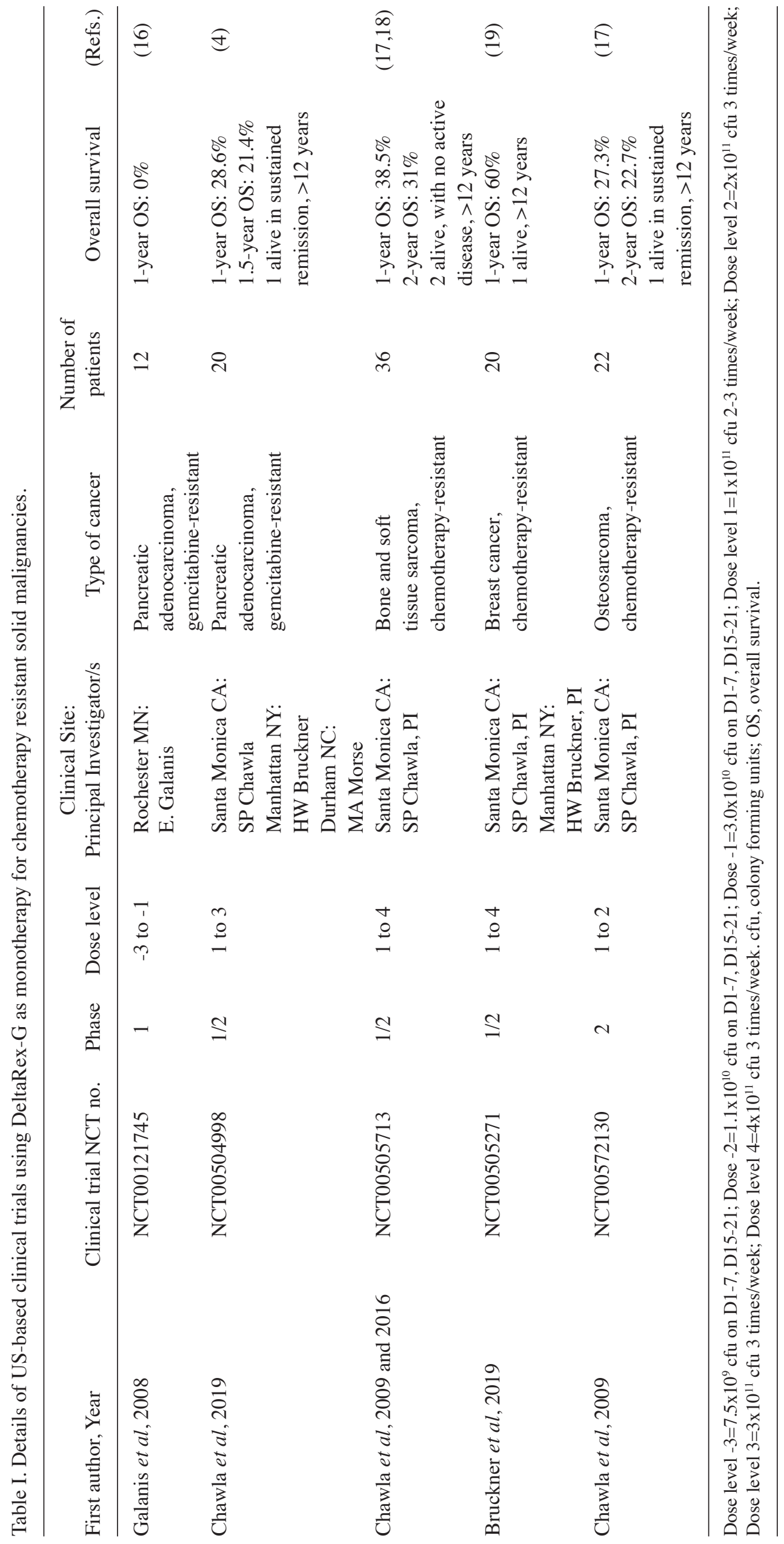


to inhibit the growth of liver metastasis in vivo in a nude mouse model of p53 mutated pancreatic cancer, likely through apoptosis-mediated pathways (14). Finally, intravenous infusions of DeltaRex-G inhibited tumor growth in vivo in a subcutaneous human xenograft model of pancreatic cancer expressing TP53 hot-spot mutations (15).

In conclusion, DeltaRex-G seeks out tumors, inhibits tumor growth and eradicates metastatic tumors and, plausibly, cancer stem cells, by precisely blocking the proliferative competence of cancer cells with CCNGl oncogene-targeted therapy for a prolonged period of time. An interesting emerging concept is that patients with advanced oncogene-addicted tumors, even those harboring TP53 mutation/loss, may still respond favorably to DeltaRex-G gene-targeted therapy, while the cytocidal CCNG1 inhibitor expressed by DeltaRex-G is itself lethal in the presence or absence of a functional p53 gene, and the inhibition of CCNG1 by complementary molecular genetic approaches may indirectly (through $\mathrm{Mdm} 2$ ) restore the tumor-suppressive function of p53, highlighting DeltaRex-G as an optimal targeted therapy for pancreatic adenocarcinoma with a prevalence of TP53 mutations (13). Studies are planned to determine whether oncogenic drivers along the CCNG1 pathway (5) could be exploited to achieve effective therapies for pancreatic adenocarcinoma, sarcoma and other solid tumors, since DeltaRex-G has achieved long-term survival ( $>12$ years) in a number of patients with chemotherapy-resistant hard-to-treat stage 4 solid as well as hematological malignancies (Table I).

\section{Acknowledgements}

The authors would like to thank Heather Gordon, Director of Operations at Aveni Foundation (Santa Monica, CA, USA) for the graphic illustrations.

\section{Funding}

No funding was received.

\section{Availability of data and materials}

The datasets used and/or analyzed during the current study are available from the corresponding author on reasonable request.

\section{Authors' contributions}

MAM, SPC and HWB were the principal investigators of the clinical trial, conducted the study and evaluated the patients' tumor responses, survival and safety, wrote parts of the manuscript, as well as reviewed and edited the final manuscript. MAM and SPC assessed the authenticity of all the raw data. TZW evaluated and provided the PET CT images, and reviewed and edited the manuscript. EMG and FLH designed the clinical protocol and informed consent, submitted the Investigational New Drug application to the US Food and Drug Administration, wrote parts of the manuscript, reviewed the published literature, oversaw the clinical trial, as well as reviewed and edited the final manuscript. All authors have read and approved the final manuscript.

\section{Ethics approval and consent to participate}

The clinical protocol was approved by the US Food and Drug Administration, the Western Institutional Review Board and the Institutional Biosafety Committee for the Cancer Center of Southern California and Bruckner Oncology, and by the Institutional Review Board and Institutional Biosafety Committee of Duke University Medical Center. Written informed consent was obtained from each patient prior to DeltaRex-G treatment.

\section{Patient consent for publication}

The patient signed a written informed consent form to use the data provided in this manuscript.

\section{Competing interests}

MAM, SPC, TZW and HWB have no competing interests. EMG and FLH are co-inventors of DeltaRex-G, including its targeted gene delivery system, which was originally developed at the University of Southern California Keck School of Medicine (Los Angeles, CA, USA) and are co-founders of Delta Next-Gene, LLC (Santa Monica, CA, USA). Patent applications are being prosecuted by Delta Next-Gene, LLC. EMG is the founder and president of the Aveni Foundation, an IRS approved 501(c)(3) public charity (Seattle, WA, USA).

\section{References}

1. American Cancer Society: Survival Rates for Pancreatic Cancer. American Cancer Society. https://www.cancer.org/cancer/ pancreatic-cancer/detection-diagnosis-staging/survival-rates. html. Acessed February 12, 2021.

2. Malone ER, Oliva M, Sabatini PJB, Stockley TL and Siu LL: Molecular profiling for precision cancer therapies. Genome Med 12: 8, 2020.

3. Von Hoff DD, Ervin T, Arena FP, Chiorean G, Infante J, Moore M, Seay T, Tjulandin SA, Ma WW, Saleh MN, et al: Increased survival in pancreatic cancer with nab-paclitaxel plus Gemcitabine. N Engl J Med 369: 1691-1703, 2013.

4. Chawla SP, Bruckner H, Morse MA, Assudani N, Hall FL and Gordon EM: A phase I/II clinical study on the safety, efficacy, and therapeutic potential of intravenous DeltaRex-G: A tumor-targeted retrovector encoding a dominant-negative cyclin G1 inhibitor for advanced pancreatic cancer. Mol Ther Oncol 12: 56-67, 2019.

5. Al-Shihabi A, Chawla SP, Hall FL and Gordon EM: Exploiting oncogenic drivers along the CCNG1 pathway for cancer therapy and gene therapy. Mol Ther Oncolytics 11: 122-126, 2018.

6. Esfahani MS, Lee LJ, Jeon YJ, Flynn RA, Stehr H, Hui AB, Ishisoko N, Kildebeck E, Newman AM, Bratman SV, et al: Functional significance of U2AF1 S34F mutations in lung adenocarcinomas. Nat Commun 10: 5712, 2019.

7. Cicenas J,Kvederaviciute K,Meskinyte I, Meskinyte-Kausiliene E, Skeberdyte A and Cicenas J: KRAS, TP53, CDKN2A, SMAD4, BRCA1, and BRCA2 mutations in pancreatic cancer. Cancers (Basel) 9: 42, 2017.

8. Kimura SH and Nojima H: Cyclin G1 associates with MDM2 and regulates accumulation and degradation of $\mathrm{p} 53$ protein. Genes Cells 7: 869-880, 2002.

9. Giono LE and Manfredim JJ: The p53 tumor suppressor participates in multiple cell cycle checkpoints. J Cell Physiol 209: 13-20, 2006.

10. Gordon EM, Ravicz JR, Liu S, Chawla SP and Hall FL: Cell cycle checkpoint control: The cyclin G1/Mdm2/p53 axis emerges as a strategic target for broad-spectrum cancer gene therapy-a review of molecular mechanisms for oncologists. Mol Clin Oncol 9: 115-134, 2018. 
11. Chen X: Cyclin G: A regulator of the p53-Mdm2 network. Dev Cell 2: 518-519, 2002.

12. Jensen MR, Factor VM, Fantozzi A, Helin K, Huh CG and Thorgeirsson SS: Reduced hepatic tumor incidence in Cyclin G1-deficient mice. Hepatology 37: 862-870, 2003.

13. Olivier M, Hollstein M and Hainau P: TP53 mutations in human cancers: Origins, consequences, and clinical use. Cold Spring Harb Perspect Biol 2: a001008, 2010.

14. Gordon EM, Liu PX, Chen ZH, Liu L, Whitley MD, Gee C, Groshen S, Hinton DR, Beart RW and Hall FL: Inhibition of metastatic tumor growth in nude mice by portal vein infusions of matrix-targeted retroviral vectors bearing a cytocidal cyclin G1 construct. Cancer Res 60: 3343-3347, 2000.

15. Gordon EM, Chen ZH, Liu L, Whitley M, Liu L, Wei D, Groshen S, Hinton DR, Anderson WF, Beart RW Jr and Hall FL: Systemic administration of a matrix-targeted retroviral vector is efficacious for cancer gene therapy in mice. Hum Gene Ther 12 : 193-204, 2001.

16. Galanis E, Carlson SK, Foster NR, Lowe V, Quevedo F, McWilliams RR, Grothey A, Jatoi A, Alberts SR and Rubin J: Phase I trial of a pathotropic retroviral vector expressing a cytocidal cyclin G1 construct (Rexin-G) in patients with advanced pancreatic cancer. Mol Ther 16: 979-984, 2008.
17. Chawla SP, Chua VS, Fernandez L, Quon D, Saralou A, Blackwelder WC, Hall FL and Gordon EM: Phase I/II and phase II studies of targeted gene delivery in vivo: Intravenous Rexin-G for chemotherapy-resistant sarcoma and osteosarcoma. Mol Ther 17: 1651-1657, 2009.

18. Chawla SP, Chawla NS, Quon D, Chua-Alcala V, Blackwelder WC, Hall FL and Gordon EM: An advanced phase 1/2 study using an XC-targeted gene therapy vector for chemotherapy resistant sarcoma. Sarcoma Res Int 3: 1024, 2016.

19. Bruckner H, Chawla SP, Liu S, Assudani N, Hall FL and Gordon EM: Phase I-II study using Rexin-G, a tumor-targeted retrovector encoding a cyclin G1 inhibitor for metastatic carcinoma of breast: A ten-year follow-up. Presented at the 2019 American Society of Gene and Cell Therapy (ASGCT) Annual Meeting. ASGCT, Washington, DC, Abstract 273, 2019.

(7) (9) This work is licensed under a Creative Commons Attribution-NonCommercial-NoDerivatives 4.0 International (CC BY-NC-ND 4.0) License. 